\title{
Rol del tutor en el desarrollo de habilidades cognitivas en alumnos con discapacidad intelectual
}

\section{Role of the tutor in the development of cognitive skills in students with intellectual disabilities}

DOI: $10.46981 /$ sfjhv2n3-008

Received in: April 1st, 2021

Accepted in: May 31th, 2021

\author{
Mg. Milagros Murillo Benavides \\ Procedencia: Universidad Católica de Santa María - Arequipa - Perú \\ Correo electrónico: milimube@ @otmail.com
}

\section{RESUMEN}

La presente investigación tuvo como objetivo diagnosticar las habilidades cognitivas en los alumnos del centro de educación básica especial Unámonos, desde el año 2013 hasta el año 2016.

Con tal objetivo se realizó una medición de las habilidades cognitivas de los alumnos del centro el año 2013 (76 estudiantes ) para posteriormente realizar una nueva medición de dichas habilidades el año 2016, luego de la aplicación de 3 años consecutivos del plan de acción individual en cada uno de los alumnos a fin de evaluar los resultados obtenidos, adicionalmente durante los años 2014,2015 se realizó una evaluación (de 23 estudiantes ),para evaluar la eficacia de la estrategia plan de acción individual.

Cabe resaltar que para la realización de la presente investigación y como parte del proceso de evaluación de las habilidades cognitivas se procedió a la creación de instrumentos especialmente diseñados y acordes a las necesidades de niños con habilidades diferentes, mismos que fueron creados por la autora, dichos instrumentos incluyen la evaluación del desarrollo integral en alumnos con habilidades diferentes en sus diferentes versiones A, B, C Y D para alumnos cuyas edades oscila entre los 3 y 28 años. La información obtenida en la presente investigación fue recogida a través de evaluaciones individuales con cada uno de los alumnos del centro, tiempo en el cual se tomaron todas las previsiones las cuales incluyen fotografías y videos de los alumnos a fin de alcanzar la mayor validez y confiabilidad del estudio.

Cabe resaltar que al finalizar la investigación se observó un incremento significativo en las habilidades cognitivas de cada uno de los alumnos especialmente en áreas tales como: secuencia de palabras, lenguaje comprensivo y nociones espaciales, áreas donde se observaron mejoras en los estudiantes de hasta un $80 \%$ alcanzándose los objetivos generales y específicos propuestos para este trabajo. La hipótesis de la investigación, "todos los alumnos, independientemente del grado de discapacidad que presenten, tienen la posibilidad de mejorar y desarrollar sus habilidades a partir de mediciones adecuadas y de estrategias pedagógicas apropiadas", fue totalmente comprobada.

Palabras clave: Habilidades cognitivas, desarrollo integral, creación de instrumentos, test: evaluación del desarrollo integral en alumnos con habilidades diferentes.

\footnotetext{
ABSTRACT

The objective of this research was to diagnose the cognitive skills of the students of the Unámonos basic special education center, from 2013 to 2016.

With such objective, a measurement of the cognitive skills of the students of the center was carried out in 2013 (76 students) to subsequently perform a new measurement of such skills in 2016, after the application of 3 consecutive years of the individual action plan in each of the students in order to evaluate the results obtained, additionally during the years 2014,2015 an evaluation was carried out (of 23 students), to evaluate the effectiveness of the individual action plan strategy.
} 
It should be noted that in order to carry out this research and as part of the evaluation process of cognitive skills, we proceeded to the creation of instruments specially designed and according to the needs of children with different abilities, which were created by the author, these instruments include the evaluation of the integral development in students with different abilities in their different versions A, $\mathrm{B}, \mathrm{C}$ and $\mathrm{D}$ for students whose ages range between 3 and 28 years. The information obtained in the present research was collected through individual evaluations with each of the students of the center, during which time all the previsions were taken, including photographs and videos of the students in order to achieve the greatest validity and reliability of the study.

It should be noted that at the end of the research a significant increase was observed in the cognitive skills of each of the students, especially in areas such as: word sequence, comprehension language and spatial notions, areas where improvements were observed in the students of up to $80 \%$, reaching the general and specific objectives proposed for this work. The hypothesis of the research, "all students, regardless of the degree of disability they present, have the possibility of improving and developing their skills based on adequate measurements and appropriate pedagogical strategies", was fully proven.

Key words: Cognitive abilities, integral development, creation of instruments, test: evaluation of integral development in students with disabilities.

\section{INTRODUCCION}

El interés por las necesidades educativas de los estudiantes con habilidades diferentes ha aumentado significativamente en las últimas décadas, convirtiéndose en la actualidad en un tema de interés;- No obstante la cantidad de información sobre dicha problemática es aún insuficiente, por lo cual surge la necesidad de desarrollar investigaciones y obtener nuevos conocimientos acerca del mismo, especialmente en lo que relacionado al trabajo e intervención integral en alumnos con discapacidad intelectual.

Por lo cual la autora se propuso lograr una comprensión integral del alumno, para lo cual resultó indispensable contar con el apoyo y la colaboración de los diferentes participantes del proceso educativo, especialmente los tutores a fin de encontrar las mejores y más eficaces herramientas para ayudar a los alumnos con discapacidad intelectual, incentivando el logro de la autorrealización personal, meta trascendental en la vida de todo ser humano.

Finalmente la autora quisiera agradecer la oportunidad brindada de poder conocer y ayudar a estos niños y jóvenes. Al ser ella misma una persona con habilidades diferentes, esta investigación le ha tocado muy de cerca, convirtiéndose en un impulso aún mayor para dar lo mejor de sí durante todo el proceso. 


\section{DESCRIPCIÓN DE LA REALIDAD PROBLEMÁTICA}

El problema en cuestión se ubica en el área de Psicología Educativa; en la especialidad de Psicología Excepcional; y en la línea o tópico de la discapacidad.

La discapacidad tiene un enfoque diverso desde el punto de vista investigativo. Como eje integrador la investigación de este tema involucra aspectos como las características de la discapacidad, los factores asociados a esta condición, las consecuencias psicosociales de la misma, la eficacia de programas que promueven el desarrollo de los alumnos, así como las características de estos y el nivel de capacitación de docentes y personal que trabaja con ellos.

Al ser un tema relativamente nuevo, particularmente en Latinoamérica, ya que normalmente se suele ver la discapacidad desde un aspecto básicamente limitante, dejando de lado todas las habilidades y fortalezas que pueden desarrollar las personas con algún tipo o grado de discapacidad, pues sin importar el grado o nivel de discapacidad todo ser humano cuenta con un potencial ilimitado, el cual puede y debe ser desarrollado y estimulado.

A través de la presente investigación se buscó en todo momento desarrollar las habilidades cognitivas de los alumnos, centrándonos en primer término en sus fortalezas y todos aquellos aspectos positivos que cada uno de nuestros alumnos posee, para a partir de allí empezar a identificar y trabajar posteriormente con sus debilidades a fin de que puedan ser superadas lo mejor posible de manera gradual y sostenida.

\section{JUSTIFICACIÓN}

El trabajo en el mejoramiento del proceso de diagnóstico en niños y jóvenes con Síndrome de Down y discapacidad intelectual permitirá no sólo tener una visión más clara y precisa de las fortalezas y debilidades de estos niños en el aspecto cognitivo, sino que también ayudará eficazmente en el desarrollo de estrategias que les permitan lograr un mayor y mejor desarrollo de sus capacidades, repercutiendo positivamente en su desarrollo integral.

\section{OBJETIVOS:}

\section{Objetivo general:}

Establecer un diagnóstico de las habilidades cognitivas de los alumnos con discapacidad intelectual a fin de identificar las estrategias óptimas que permita el desarrollo de las habilidades del alumno.

\section{Objetivos específicos:}

- Diagnosticar de manera precisa, la problemática de los alumnos con discapacidad intelectual 
- Estimular el desarrollo de las fortalezas de los alumnos con discapacidad intelectual en el aspecto cognitivo, para ayudarlos a alcanzar un desenvolvimiento de mayor funcionalidad en su vida cotidiana.

- Capacitar y asesorar a los profesores de alumnos con discapacidad intelectual en las diversas inquietudes que puedan presentar en su trabajo con dichos alumnos a fin de determinar las mejores estrategias de intervención.

\section{CONCEPTOS BÁSICOS:}

1. Discapacidad: trastorno caracterizado por limitaciones funcionales que impiden un desarrollo normal como consecuencia de una perturbación sensorial o física, de una dificultad en el aprendizaje o también de una adaptación social deficiente (Heward, 1998). En el Centro de Educación Básica Especial donde se aplicaron los instrumentos y se llevó a cabo la investigación, se encuentran fundamentalmente alumnos con discapacidad de tipo intelectual, que se caracteriza por tratarse de un trastorno de tipo cognitivo y que incluye limitaciones en la conducta adaptativa (Deutsch, 2003). La mayoría de los estudiantes con discapacidad intelectual participantes del estudio presentaban como diagnóstico Síndrome de Down, si bien también hay alumnos que tienen parálisis cerebral y autismo, entre otros.

2. Habilidades cognitivas: hacen referencia a una serie de procesos del pensamiento que permiten adquirir conocimientos, integrando la información recibida por medio de los sentidos en forma coherente. En el proceso de evaluación se incluyeron áreas como:

- Lenguaje comprensivo: es la habilidad para discernir lo que se dice, comprender órdenes y consignas e ideas (Báez, 2011).

- Esquema y preparación corporal: incluye la capacidad para reconocer el propio cuerpo y distinguirlo del de los demás, igualmente incluye la habilidad para identificar las partes del cuerpo humano;

- Memoria: es la capacidad de retener información a lo largo del tiempo. Existen varios tipos de memoria: memoria a corto plazo y memoria a largo plazo. En el proceso de evaluación se ha considerado la memoria a corto plazo;

- Atención y concentración: implica dirigir la atención y mantenerla enfocada en aspectos o situaciones y vivencias específicas de manera sostenida, durante un tiempo determinado;

- Coordinación: consiste en el uso de diferentes partes del cuerpo de manera organizada a fin de ejecutar acciones específicas. El desarrollo de la coordinación motora es base para la adquisición de nuevos aprendizajes y destrezas. Durante la evaluación se hizo énfasis en la 
examinación de la coordinación motora fina, incluyendo el enhebrado, ensartado, la prensión y la precisión.

- Razonamiento: implica la capacidad para resolver problemas y deducir conclusiones e incluye la identificación de secuencias lógicas y la abstracción;

- Percepción visual: es el proceso mental de recepción e interpretación de la información que se recibe a través de estímulos visuales.

- Ubicación temporal: entendida como la capacidad para identificar el momento en que una persona se encuentra y reconocer la secuencia de pasado, presente y futuro de los eventos. Permite que la persona pueda conectarse con su entorno e interactuar con el mismo de manera satisfactoria.

\section{METODOLOGÍA}

El estudio, de carácter descriptivo (Salkind, 1999) fue realizado con los 90 alumnos del Centro de Educación Básica Especial UNÁMONOS de Arequipa, cuyas edades oscilan entre los 3 y 25 años; valorando los resultados desde el enfoque mixto, combinando enfoques cuantitativo y cualitativo (Hernández, Fernández y Baptista, 2010). El enfoque cuantitativo recoge la información y le asigna valores numéricos y de cantidades, mientras que el enfoque cualitativo recoge la información sin medirlos de forma numérica, interpretándolos en base a las cualidades mismas de los datos recogidos. Debe señalarse que, aunque se trata de un enfoque mixto, existe cierta predominancia de la perspectiva cualitativa en el presente proceso de evaluación.

\section{DESARROLLO DE INSTRUMENTOS}

Antes de iniciar la investigación, se revisaron diferentes tests y cuestionarios usados tradicionalmente, encontrándose que tales instrumentos no se adecuaban completamente a las necesidades de personas con habilidades diferentes. Por este motivo, la autora consideró necesaria la adaptación y creación de instrumentos para la medición de habilidades cognitivas, respectivamente. Esta herramienta de evaluación, diseñada acordes a la realidad de los alumnos con discapacidad intelectual, incluyen el uso de imágenes y demostraciones además de las indicaciones de carácter verbal. Esto facilitó significativamente el proceso evaluativo debido a que muchos de los estudiantes todavía no han desarrollado un lenguaje expresivo completamente fluido. El uso de instrumentos creados especialmente para las necesidades del estudiante ayudó a conectar con mayor facilidad con los conocimientos previos del niño y joven, lo que a su vez permitió identificar de manera más clara sus fortalezas y debilidades. 
Para la investigación se crearon dos instrumentos, validados a través de la prueba piloto inclusa y el juicio de expertos ${ }^{1}$ :

1. La evaluación del desarrollo integral en alumnos con habilidades diferentes (DIAHD) ${ }^{2}$ que consta de cuatro formas principales ${ }^{3}$ :

- Forma A: para niños de 3 a 6 años;

- Forma B: para niños de 7 a 12 años;

- Forma C: para niños de 13 a 25 años

- Forma D: para alumnos con discapacidad intelectual severa.

Cada forma está conformada por una serie de áreas destinadas a evaluar, a través de diversos ítems, las diferentes habilidades cognitivas del alumno. Es importante señalar que no todas las áreas se encuentran presentes en todas las Formas, debido a que se ha tenido el nivel de desarrollo en el que se encontraría el estudiante de acuerdo a su edad así como el grado de discapacidad que presenta, y existen algunas áreas que se desarrollan a medida que el alumno va creciendo.

\section{VALIDACIÓN DE INSTRUMENTOS}

Para este proceso, se emplearon los siguientes procedimientos:

1. Prueba piloto inclusa: este procedimiento consistió en la aplicación de los instrumentos a varios de los estudiantes con la finalidad de verificar si la estructura del instrumento - incluyendo aspectos con la redacción y la elección de ítems - resultaba comprensible por los alumnos y medían aquello que estaban destinados a medir. El carácter de "inclusa" asignado a esta forma de prueba piloto se debe a que la información obtenida durante las sesiones de evaluación forma parte del análisis de las habilidades cognitivas.

2. Juicio de expertos: este procedimiento permitió validar los instrumentos de acuerdo a los siguientes criterios:

- Claridad y precisión: los ítems están redactados en forma clara y precisa y sin ambigüedades,

- Coherencia: el contenido de los ítems guarda relación estrecha con las áreas evaluadas,

- Validez: los ítems miden lo que pretenden medir,

- Organización: las áreas e ítems se encuentran siguiendo una secuencia lógica,

- Confiabilidad: los resultados obtenidos a través de los instrumentos, son consistentes y coherentes,

- Metodología: la metodología corresponde con el enfoque de la investigación,

\footnotetext{
${ }^{1}$ Juicio de expertos: Dr. Charles Portilla Revollar, Dr. Nicolás Paredes Carpio, Dr. Abel Tapia Fernández

${ }^{2}$ Creado por Murillo, M. (2013)

${ }^{3}$ Derechos reservados INDECOPI, registros 00355-2014, 00306-2014
} 
- Pertinencia: los instrumentos son adecuados para el objetivo del estudio,

- Objetividad: los ítems están redactados según conductas observables y medibles,

- Marco de referencia: los instrumentos tienen en cuenta las características de la población ,

- Extensión: el número de ítems en los instrumentos, no resulta excesivo,

- Inocuidad: los ítems no representan riesgo alguno para las personas que responden a la evaluación,

- Consistencia: los instrumentos poseen respaldo teórico - práctico

Los instrumentos fueron revisados por los siguientes expertos:

- Dr. Abel Tapia, experto en investigación,

- Dr. Charles Portilla, Ph.D., experto en desarrollo infantil, problemas en el desarrollo y psicología de excepcionalidad,

- Dr. Nicolás Paredes, experto en educación especial e investigación.

\section{ESTRATEGIA DE APLICACIÓN}

Para la evaluación de las habilidades cognitivas, el DIAHD fue aplicado de forma individual. El estudiante respondía los diferentes ítems del test. Las indicaciones eran explicadas con calma para que el alumno pudiera comprenderlas, repitiéndolas en caso de que no fueran comprendidas por completo la primera vez. Existía cierta flexibilidad en la forma en cómo el estudiante podía expresar sus respuestas - bien sea indicando el nombre del objeto que veía, o haciendo un sonido que hiciera referencia al reconocimiento y comprensión del ítem -. El tiempo aproximado de aplicación es de 45 minutos, aunque podía extenderse, en una sola sesión.

\section{VALORACIÓN DE RESULTADOS}

1. Habilidades cognitivas: el DIAHD clasifica las puntuaciones obtenidas en las siguientes tres categorías:

- Desarrollo satisfactorio: el desarrollo de estas habilidades se encuentra bien encaminado y desarrollándose de manera adecuada,

- En proceso de desarrollo: significa que las habilidades del alumno se encuentran en plena formación,

- Necesita mejorar: las habilidades del educando se encuentran todavía en un nivel incipiente de su resultado. 


\section{RESULTADOS Y GRÁFICOS}

A continuación se presentan algunos de los gráficos más representativos de la investigación.

RESULTADOS GENERALES NIÑOS 4 AÑOS (2013 -2016)

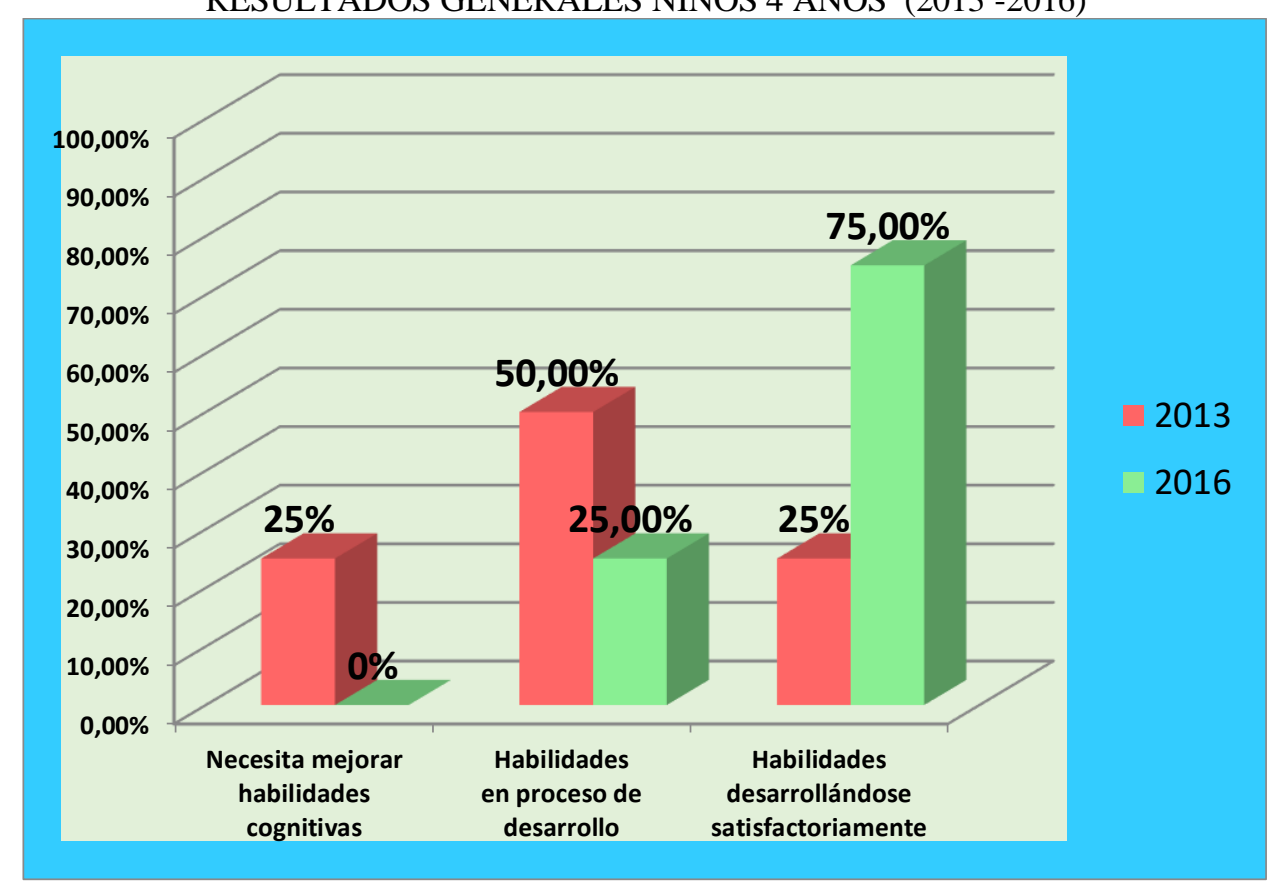

RESULTADOS GENERALES 6 AÑOS (2013-2016)

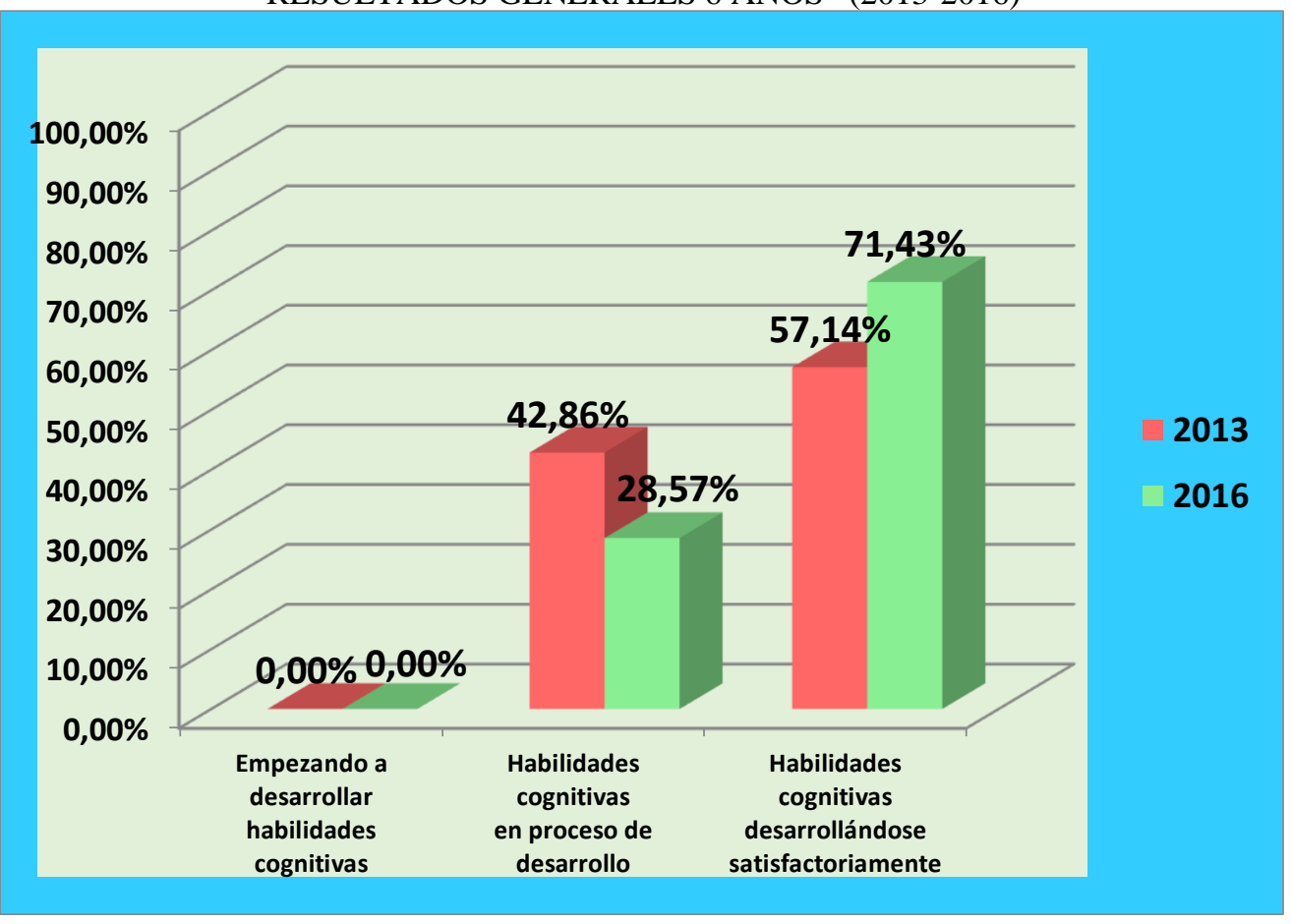


RESULTADOS GENERALES 15 AÑOS (2013 - 2016)

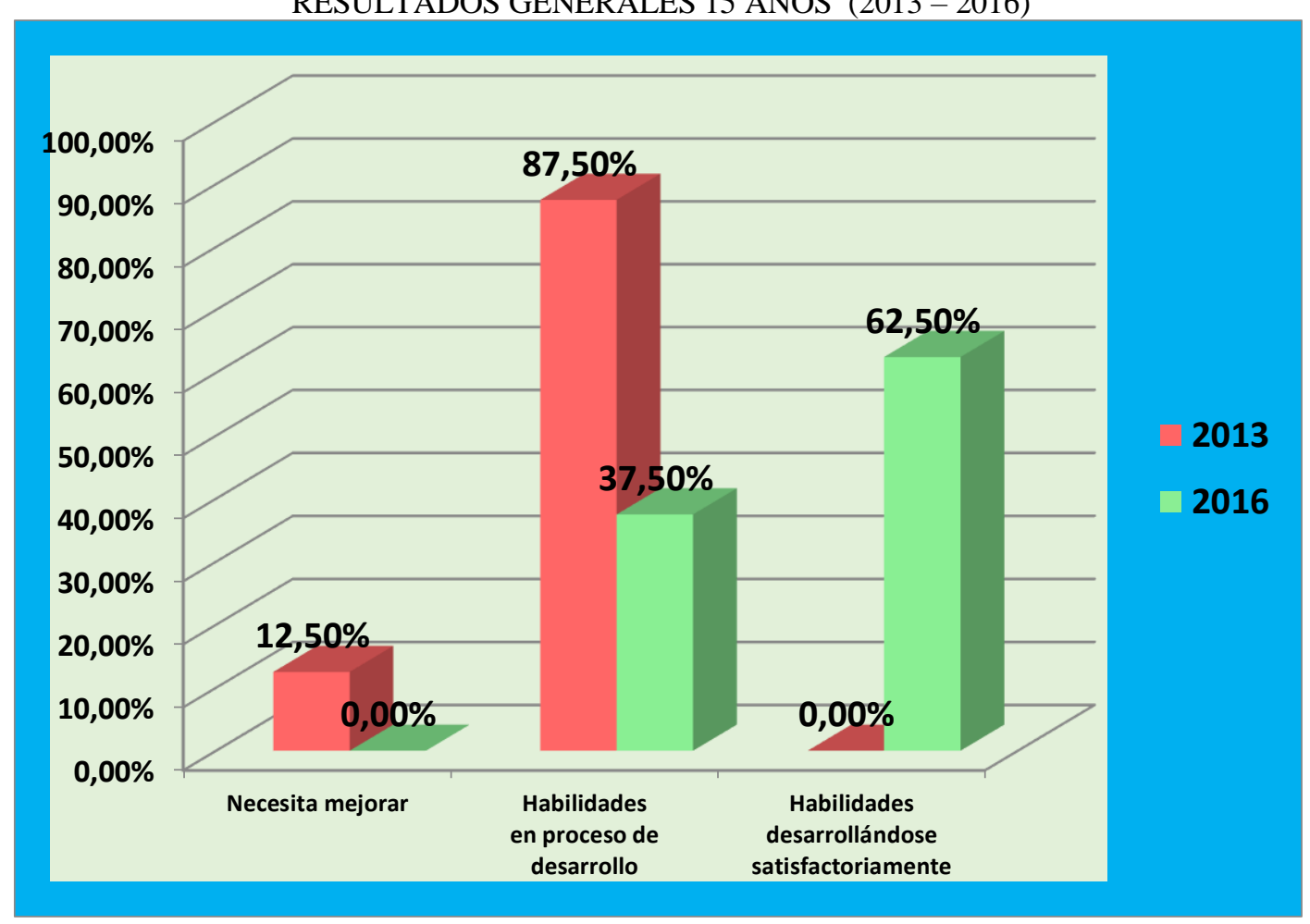

RESULTADOS GENERALES 18 AÑOS (2013 - 2016)

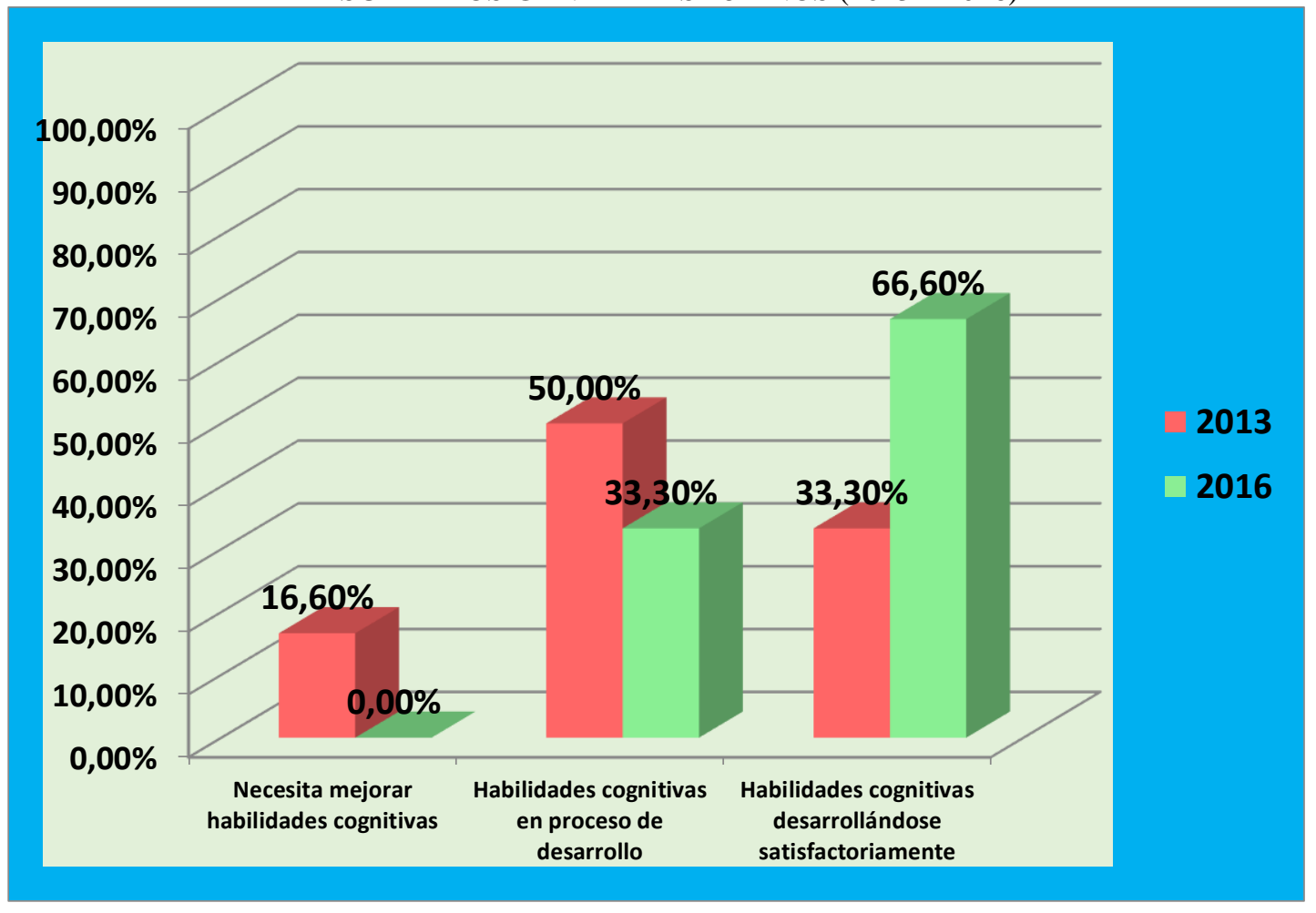


RESULTADOS GENERALES ALUMNOS CON DISCAPACIDAD SEVERA (2013 - 2016)

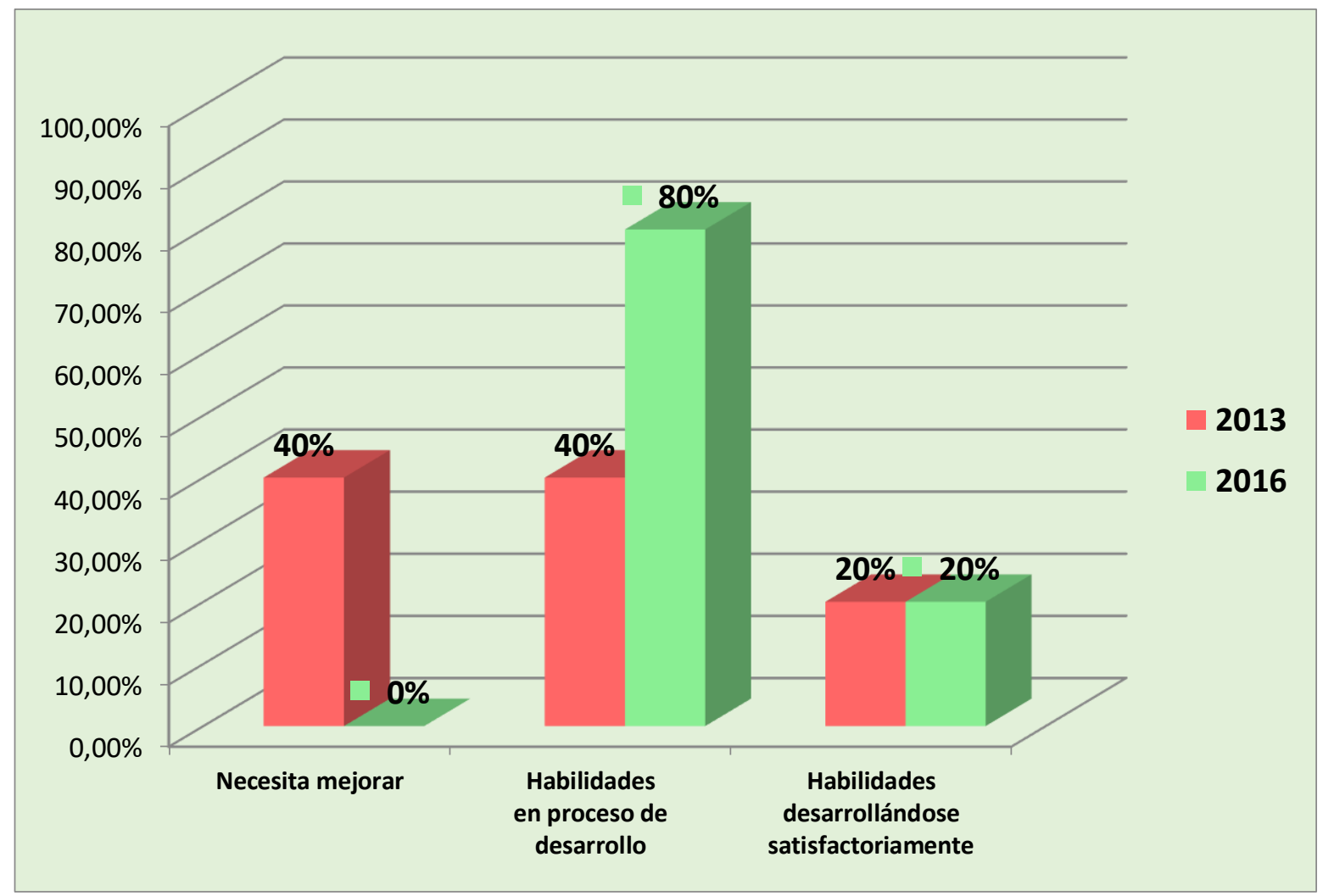


HABILIDADES COGNITIVAS:

GRUPOS EVALUADOS ANUALMENTE EN 3 GRUPOS

NIÑOS DE 7, 11 y 14 años

RESULTADOS GENERALES NIÑOS 7 AÑOS (2013)

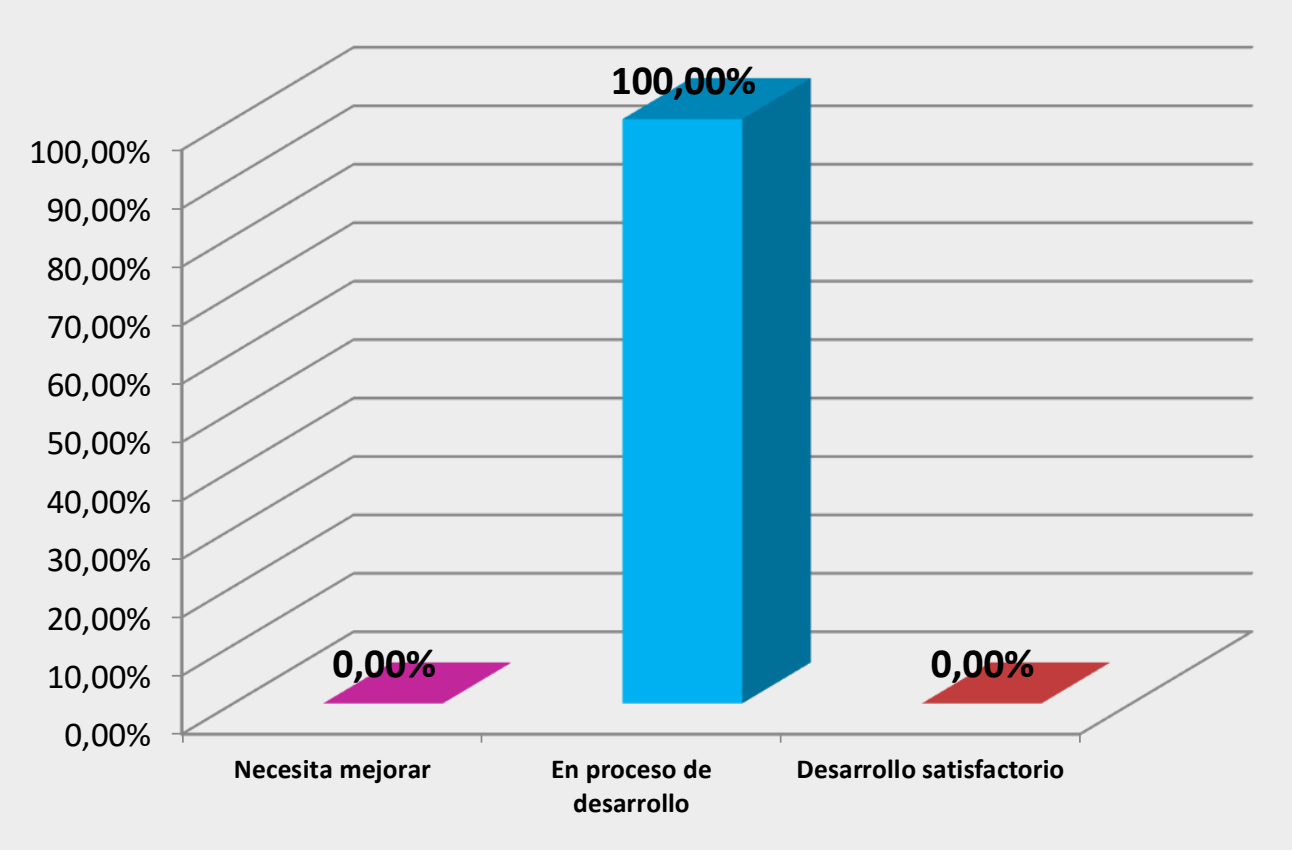

RESULTADOS GENERALES NIÑOS 7 AÑOS (2014)

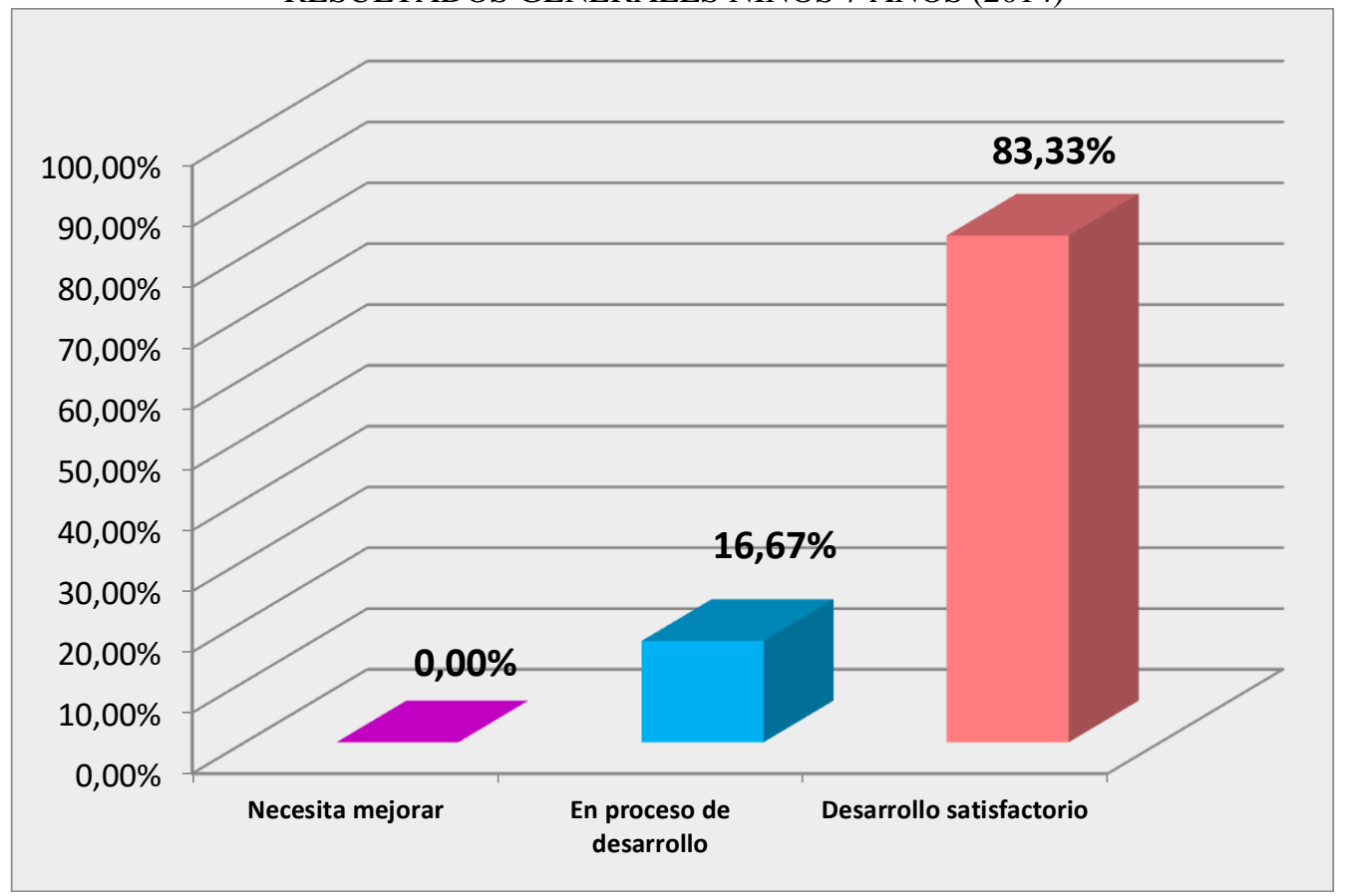


RESULTADOS GENERALES NIÑOS 7 AÑOS (2015)

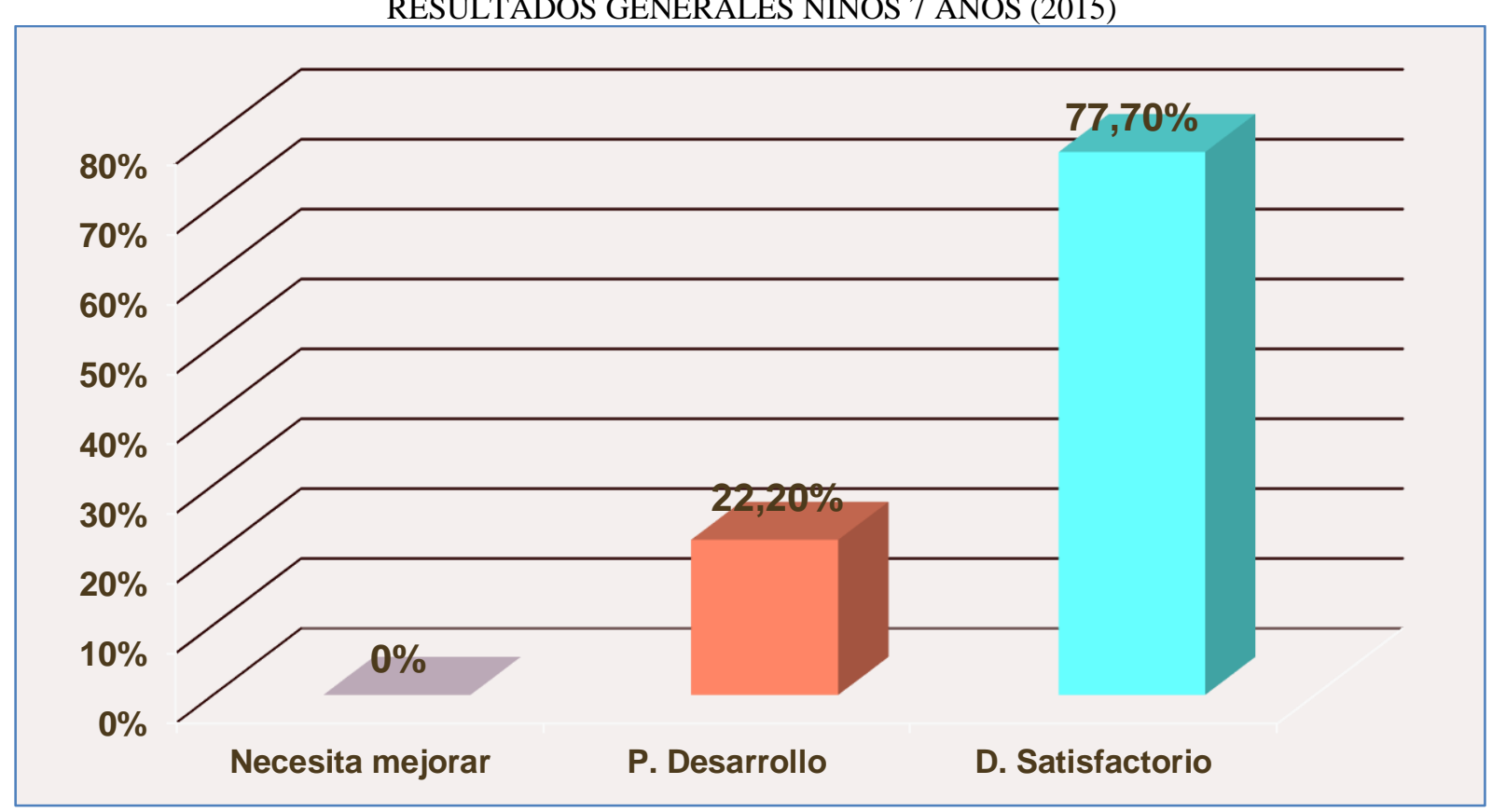

RESULTADOS GENERALES NIÑOS 7 AÑOS (2016)

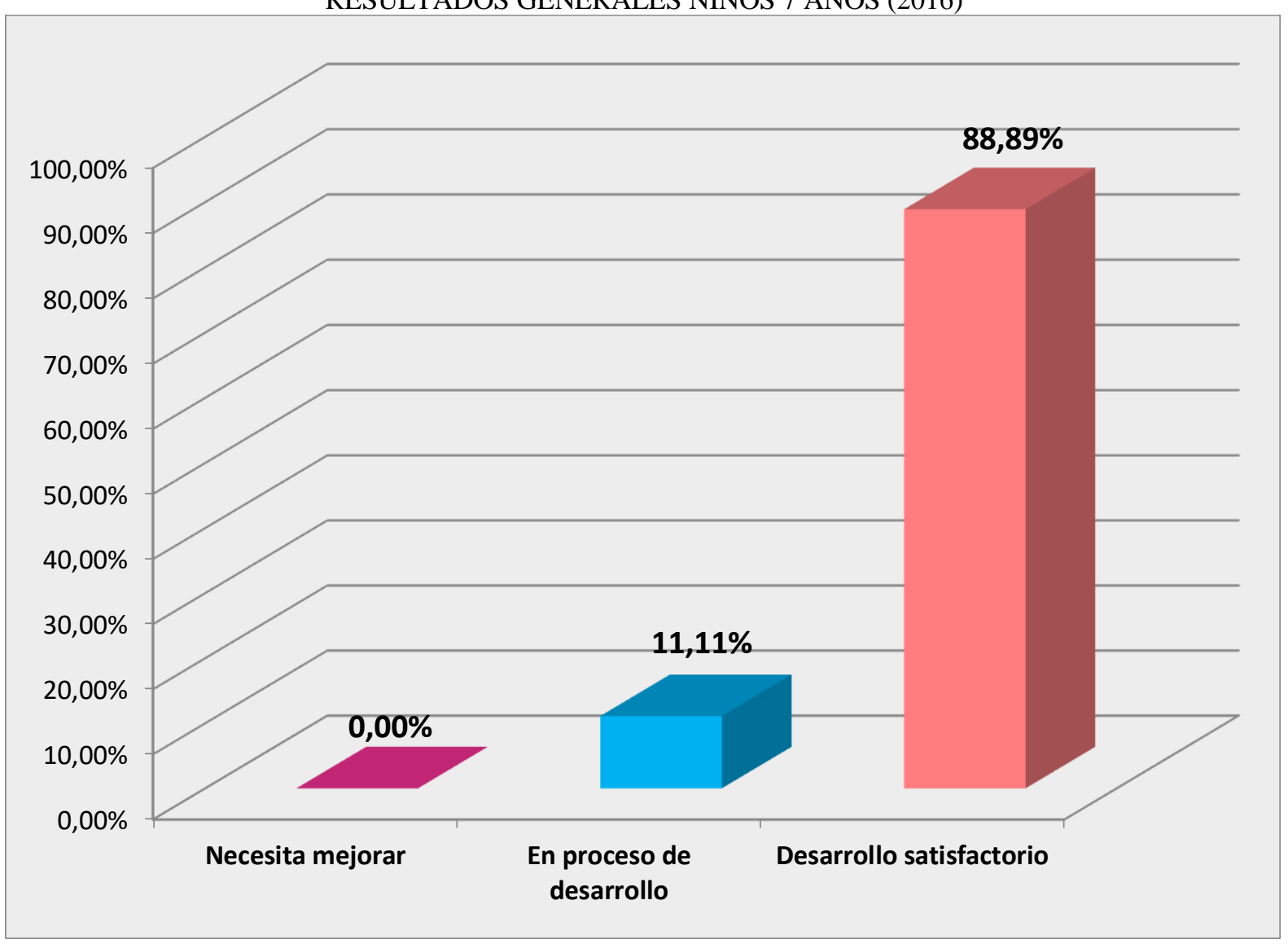




\section{CONCLUSIONES}

- En la totalidad de los 11 grupos evaluados, se observan mejoras en el área cognitiva, siendo las áreas donde se puede observar mayor avance las relacionadas a lenguaje comprensivo, secuencia de palabras, números y cantidades, y ubicación temporal.

- En los grupos donde se realizó la medición anual, la mejora en los alumnos ha sido de un 100\% en varias de las áreas entre las que pueden destacarse: lenguaje comprensivo, nociones espaciales y ubicación temporal.

- En todos los grupos se observaron mejoras significativas en su desarrollo de habilidades cognitivas, lo cual evidencia que el trabajo sistemático y constante, genera cambios, aún en los casos de discapacidad intelectual severa y multidiscapacidad. 


\section{REFERENCIAS}

Deutsch, D. (2003). Bases psicopedagógicas de la educación especial, 4ta. Ed. Madrid: Prentice Hall.

Gallego, J. \& Rodríguez, A. (2012). Bases teóricas y de investigación en educación especial. Madrid: Editorial Pirámide.

Hernández, R; Fernández, E. \& Baptista, P. (2010). Metodología de la investigación, 5ta. Edición. México, D.F.: McGraw Hill.

Heward, W. (1998) Niños excepcionales: una introducción a la educación especial. 5ta. Ed. Madrid: Prentice Hall.

Morales, G. \& López, E. (2006) El síndrome de down y su mundo emocional. México, D.F.: Editorial Trillas.

Núñez, B. (2008). Familia y discapacidad. Buenos Aires: Lugar Editorial.

Paredes, N., Portilla, Ch. \& Roberts, S. (2005) Curso de investigación de tesis. Universidad Católica de Santa María, Arequipa.

Santrock, J. (2007) Desarrollo infantil. 11va. Ed.México D.F.: McGraw Hill. 